\title{
Enhanced on Demand ECRSEP Protocol using Improved Cluster Head Selection
}

\author{
Harpinder Kaur \\ Department of Computer Science \\ Guru Nanak Dev University \\ Amritsar
}

\author{
Sandeep Waraich \\ Department of Computer Science \\ Guru Nanak Dev University \\ Amritsar
}

\begin{abstract}
Wireless Sensor network consists of large amount of low power sensor nodes generally deployed in hostile and harsh environment to sense, collect and transmit data to the sink situated at some distance. Clustering has been widely studied to enhance the lifetime of WSN by reducing the number of packet transmission. In clustering, the nodes selected as cluster head, often suffer from high overload and thus consume more energy. In this paper, we have proposed a new on demand Energy Consumption rate stable election protocol for heterogeneous WSN that uses an improved cluster head selection criteria. The improved selection criterion utilizes average remaining energy and average distance of each node which is not considered in existing protocols. Furthermore the proposed protocol being reactive improves the stability period and network lifetime. The experimental analysis depicts that the proposed algorithm has shown a significant improvement over ECRSEP.
\end{abstract}

\section{Keywords}

On demand, ECRSEP, clustering, heterogeneous, WSN, wireless, sensor, network.

\section{INTRODUCTION}

Wireless Sensor Networks (WSN) have been attracting many researchers because it has wide applications for monitoring application and data collections [1]. Network lifetime is a critical issue in WSN as the sensors being deployed have limited power. Clustering is an efficient approach towards optimized utilization of available power of sensors. Clustering techniques in wireless sensor networks aims at gathering data among groups of nodes, which elect leaders among themselves [10]. The leader or cluster-heads has the role of aggregating the data and reporting the data to the BS. This scheme reasonably improves energy usage of each node and reduced communication cost.

The clustering algorithms can be classified on the basis of homogeneity and heterogeneity of nodes. In homogeneous networks, all nodes have the same characteristics, hardware and processing capabilities In this case every sensor can become a $\mathrm{CH}$. Moreover, the $\mathrm{CH}$ role can be periodically rotated among the nodes in order achieve better load balancing and more uniform energy consumption. In heterogeneous sensor networks, there are generally two types of sensors, sensors with higher processing capabilities and complex hardware and common sensors, with lower capabilities, used to actually sense the desired attributes in the field.

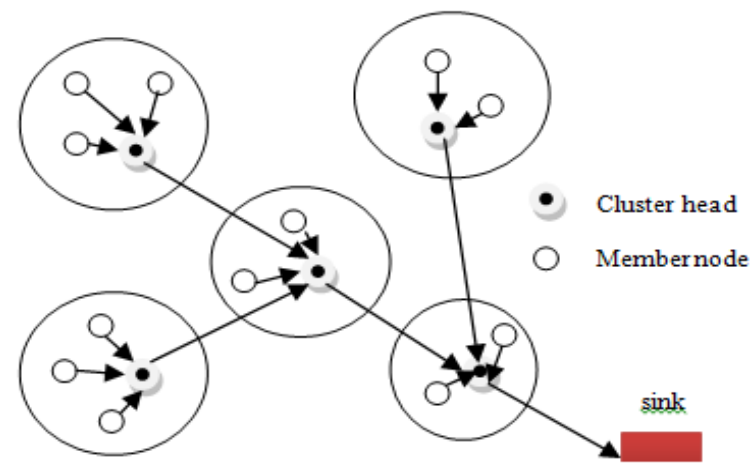

Figure:1 Data communication in Clustered Network

With purely static clustering in a homogeneous network, it is evident that $\mathrm{CHs}$ will be overloaded with long range transmissions to the remote sink, and extra processing is necessary for protocol co-ordination and data aggregation. WSN faces a problem that $\mathrm{CHs}$ dies before other nodes. However, to ensure that all nodes die at about the same time when system expires, minor amount of residual energy is wasted. One method to ensure is rotating the role of a cluster head periodically and randomly over all the nodes. The downside of role rotation and using a homogeneous network is that all nodes should be capable of act as $\mathrm{CH}$, therefore should require necessary hardware capabilities. On the other hand, in heterogeneous sensor network, two or more different types of sensor nodes in terms of different energy are used. The nodes with higher energy have more probability to be selected as cluster head. The problem area is that extra energy and complex hardware can be embedded in few $\mathrm{CH}$ nodes, therefore reducing hardware cost of the entire sensor network.

\section{RELATED WORK}

The first clustering protocol proposed for WSNs was LEACH [2]. It is a dynamic, hierarchical, probabilistic, distributed and one-hop protocol. Its major objectives were to improve the lifetime of WSNs by evenly distributing the energy consumption among all the nodes of the network and to reduce the energy consumption in the network nodes by performing data aggregation. Recently, there have been lots of other clustering techniques which are mostly variants of LEACH protocol with slight improvement and different application scenarios. PEGASIS [3], HGMR [4], UCMR [5] and HCC [6] are all homogeneous clustering techniques proposed with the objective of minimizing energy usage, while extending network life time. 
SEP [7] is based on two level heterogeneity and the $\mathrm{CH}$ election in SEP is on the basis on weighted election probability. A fraction $m$ advanced nodes in total of $n$ nodes is provided with an additional energy factor $\alpha$. So, the stability period is increased due to advance nodes, however $\mathrm{CH}$ selection is done in the same way as in LEACH. ESEP is an extension of SEP that considers three types of nodes in discussed in [8]. In [9] DEEC estimates ideal value of network life time is used to compute reference energy that each node expends during a round.

SEP is based on weighted election probabilities assigned to each node to become $\mathrm{CH}$ according to their initial energy. The rotating epoch and election probability is directly correlated with initial energy of nodes instead of residual energy of the nodes. Advance nodes more frequently becomes $\mathrm{CHs}$, it may happen after some rounds the energy of advance nodes becomes less than normal nodes. To overcome this drawback, a new $\mathrm{CH}$ selection scheme known as ECRSEP is introduced in [10] for SEP based on ECR of each node. By using this criteria SEP increases stability and lifetime of network.

In this paper a new enhanced on demand ECRSEP protocol with improved cluster head selection criteria is given which is reactive in nature. The proposed protocol considers average remaining energy and average distance of each node while selecting cluster head that was neglected in existing protocols. The simulation results shows that the given protocol is efficient than the existing ECRSEP in terms of network lifetime and throughput.

In section 3, the power consumption and network model is provided. Enhanced on demand ECRSEP protocol is given in section 4 followed by simulation results in section 5 . At the end the work is concluded in section 6 .

\section{POWER CONSUMPTION AND NETWORK MODEL}

In radio model given in ECRSEP, energy dissipates $E_{\text {elec }}=$ $50 \mathrm{~nJ} / \mathrm{bit}$ to run receiver and transmitter circuitry and $E_{a m p}$ $=100 \mathrm{pJ} / \mathrm{bit} / \mathrm{m} 2$ for transmitter amplifier. The equations that is used to calculate the receiving cost and transmitting cost for $k$ bit message and distance $d$ is modeled in [10] is as shown in below:

\section{Transmitter Energy}

$$
\begin{aligned}
& E T(K, d)=E T-\text { elect }(k)+E T-a m p(k, d) \\
& E T(K, d)=\left(E_{\text {elect }} \times K\right)+\left(E_{\text {amp }} \times k \times d 2\right)
\end{aligned}
$$

Receiving Energy

$$
\begin{aligned}
& E R(K)=E R-\operatorname{elec}(K) \\
& E R(K)=E_{\text {elec }} \times K
\end{aligned}
$$

The network model for ECRSEP assume that $N$ sensor nodes are deployed within a $M \times M$. The network is deployed into clustering hierarchy. Every cluster has a $\mathrm{CH}$, responsible to directly transmit data to Sink. We suppose that our network is stationary.

In our network we have also considered two level of heterogeneity as in ECRSEP in terms of energy. In heterogeneous networks, there are two types of sensor nodes, i.e., normal nodes and advance nodes. $E_{O}$ is initial energy of normal nodes and $m$ is fraction of advanced nodes. Advanced nodes have $\alpha$ times more energy than normal nodes. So $m N$ advanced nodes having initial energy $E o(1+\alpha)$ and $(1-m) N$ normal nodes having initial energy $E o$.

The initial energy Eo of two levels heterogeneous network is given in [10] as:

$E_{\text {total }}=N(1-m) E_{o}+N m E_{o}(1+\alpha)=N E_{o}(1+\alpha m)$

So, two level heterogeneous networks have am times more energy than homogeneous network.

\section{ENHANCED ON DEMAND ECRSEP PROTOCOL}

In enhanced ECRSEP, the cluster head selection is made on the basis of average remaining energy of node and average distance between node and base station. Mathematically, the average distance is calculated as:

D_average $=$ sum $/ n$

The remaining minimum energy level which is required to consider a node for cluster head is given as:

$x=s(i) . E^{*} n * 0.05 / E \_$total

\subsection{Improved Cluster head selection}

In ECRSEP, the cluster head is selected on the basis of energy consumption rate (ECR). In two level heterogeneous networks, $p_{\text {opt }}$ is replaced by weighted probabilities for advance and normal nodes as modeled in [10] as:

$p_{a d v}=\frac{p_{o p t} \frac{E(i)-E(r)}{r-1}}{1+\alpha m}$

$p_{\text {nrm }}=\frac{p_{\text {opt }}(1+\alpha) \frac{E(i)-E(r)}{r-1}}{1+\alpha m}$

The modified ECRSEP utilizes the distance of node from base station and average remaining energy of nodes while selecting the optimized cluster head that is proven to affect the network lifetime and throughput reasonably.

In the initial round, the advance nodes have higher probability to become cluster head and in successive rounds the cluster head selection is done through threshold function $(\mathrm{T})$ which is computed from improved probability function.

The new improved probability function $\mathrm{P}(\mathrm{i})$ which utilizes average remaining energy and distance of nodes from base station is given by $\mathrm{P}(\mathrm{i})$;

$P(i)=\alpha * f u n D+(1-\alpha) * f u n E$

Where, funD is the average distance function calculated as:

funD $=\frac{0.1}{1+\exp \left(0.5 *\left(D_{\text {average }}-\sqrt{\left.\text { xarea } * \frac{\text { yarea }}{n}\right)}\right)\right.}$

and funE is the average remaining energy function given by:

fun $E=\frac{0.1}{1+\exp ((-120) *(x-0.05))}$

The improved threshold function can be obtained from the above probability function i.e;

$T(i)=\frac{P(i)}{1-P(i) * \bmod (r, \operatorname{round}(1 / P(i)))}$

If the random value selected is less than or equals to the threshold function $(\mathrm{T})$ then the corresponding node is chosen as cluster head. 


\subsection{On Demand Data Transmission}

Further improvement is made by introducing the concept of on demand transmission inspired from TEEN [12]. Instead of sending data regularly, the data packets are transmitted when the value of sensed attribute (temperature, pressure etc.) equal or increase the predefined hard threshold value (HT). HT is an absolute value of sensed attribute beyond which node will transmit data to $\mathrm{CH}$.

The current value (CV) of first broadcast is stored in an interior variable in the sensor node called Sensed Value (SV). It decreases the amount of broadcasts. Now the sensor nodes will again communicate the packets in identical cluster time when the difference of the $\mathrm{CV}$ and $\mathrm{SV}$ is more than the ST i.e. if the CV varies from SV by a quantity equivalent to or more than SV, then it supplementary decrease the number of communications.

If $\mathrm{CV}>\mathrm{HT}$

$\mathrm{SV}=\mathrm{CV}$;

If $S V-C V>=S T$;

S(i).E=S(i).E-DATA_PCKT* $\left(\mathrm{P} 0 *\left((\text { dis } * 0.001)^{\wedge} 1.5\right)^{*}\left(\mathrm{a}^{\wedge}(\mathrm{dis} * 0.001)\right)+\mathrm{EDA}\right)$ -

ENERGY.Broad;

Figure:2 Pseudo code for on demand transmission

\subsection{SIMULATION RESULTS}

We evaluate performance of our protocol by using MATLAB. We arrange a WSN with $N=100$ nodes that are distributed randomly in $100 \mathrm{~m} \times 100 \mathrm{mfield}$. We assume in our simulations that sink is at center of sensing region. To compare performance of ECRSEP with other protocols, effect of interference and signal collision is not considered in wireless channel. Our goal is to compare performance of enhanced on demand ECRSEP with ECRSEP protocol on basis of throughput and the longevity of network.

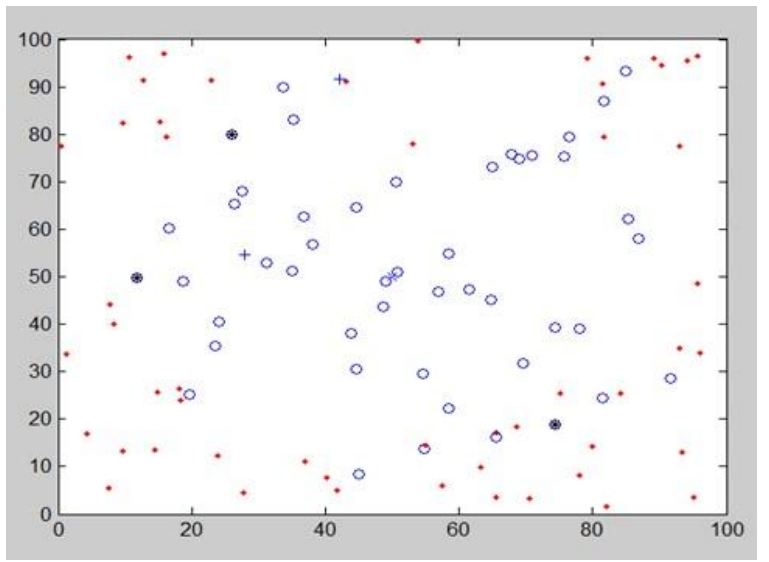

Figure:3 Initial configuration of WSN

We use following parameters in our simulation. Eelect $=$ 50nJ/bit, EDA $=5 \mathrm{~nJ} / \mathrm{bit} / \mathrm{message}, \_f s=10 \mathrm{pJ} / \mathrm{bit} / \mathrm{m} 2, \ldots m p=$ $0.0013 \mathrm{pJ} / \mathrm{bit} / \mathrm{m} 4$, Eo $=0.5 \mathrm{~J}, K=4000$, Popt $=0.1, n=100, \alpha$ $=0.5, m=0.1, H T=100, S T=0, \operatorname{minf} q=50$, and $\max q=200$.

By performing simulations in MATLAB, it is observed that, Enhanced on demand ECRSEP has enhanced stability period than all other protocol and network life for ECRSEP was increased as compared to existing ECRSEP.
Figure : 4(a) and 4(b) shows that the number of data packets transmitted to base station and cluster head respectively are greater in our proposed protocol than existing ECRSEP. It can be clearly seen that the data transmission is being carried out till 787 in proposed protocol whereas it limits in ECRSEP till 567. So we can say that the given improved on demand ECRSEP protocol increases throughput of a WSN.

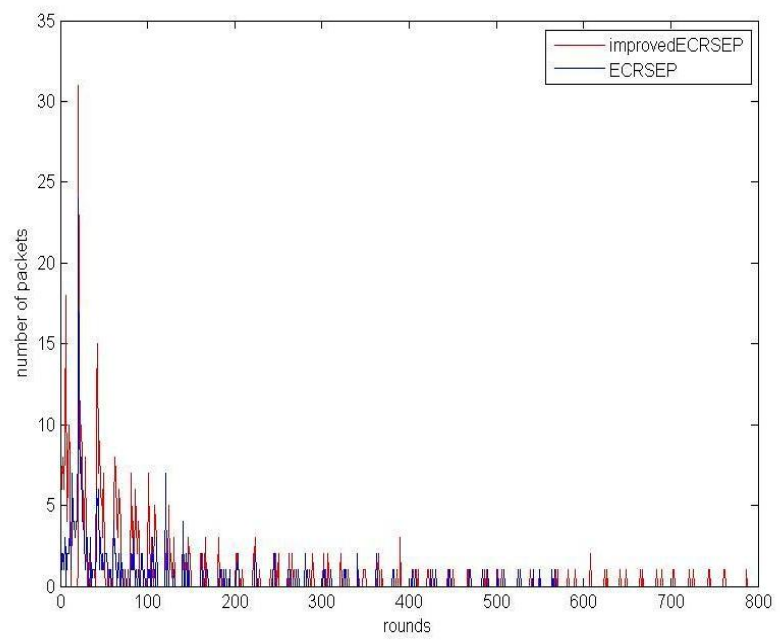

Figure:4 (a) number of packets transmitted to BS

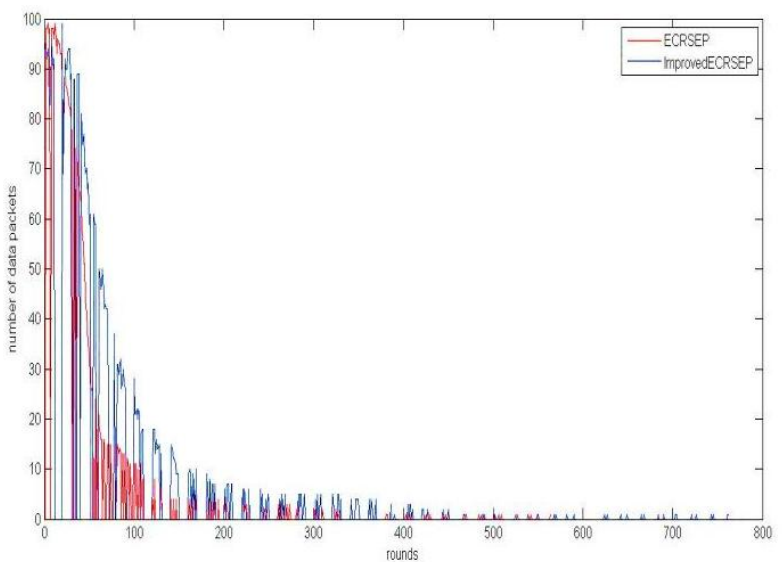

Figure:4(b) Packet send to cluster head

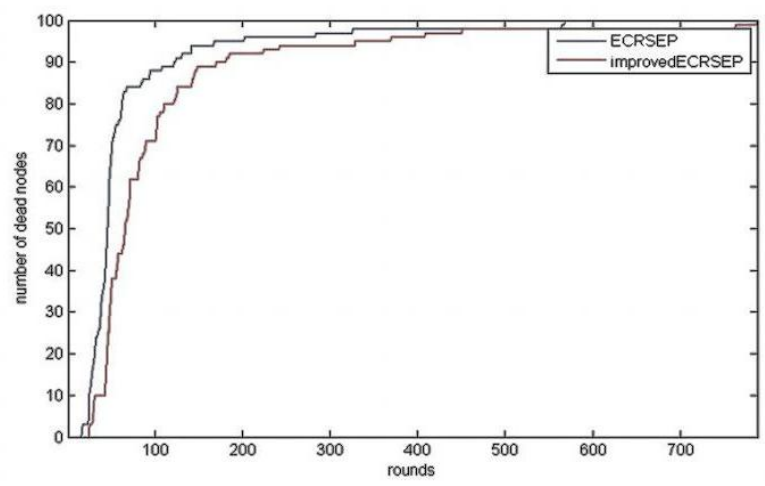

Figure:5 Network Lifetime 
Network lifetime is generally measured in terms of first node dead time or last node dead time. We have compared the performance of ECRSEP and enhanced On Demand ECRSEP under similar parameters. From figure:5, it can be seen that in ECRSEP, the first node died at round 15 and the count increases sharply to75 till round 50 approximately followed by gradual increase in dead nodes. After stable count for some rounds, the last node dies at round 567. The Enhanced On demand ECRSEP protocol shows improvement in approximately every round. The first node dies in round 24 and the last node dies in round 787 . Hence we can say that lifetime of network is increased in our proposed algorithm.

\section{CONCLUSIONS}

This research work has proposed a new Enhanced On Demand ECRSEP protocol for two-tier clustered heterogeneous network where the advanced nodes elect themselves as cluster heads for the initial round and the cluster head selection in successive rounds is done on the basis of average remaining energy and the average distance of the node. A node is chosen as cluster head if the random value is less than or equal to the threshold function. Furthermore the proposed protocol being reactive improves the stability period and network lifetime. The experimental analysis depicts that the proposed algorithm has shown a significant improvement over ECRSEP.

\section{REFERENCES}

[1] AA Abbasi, and M. Younis, " A Survey on Clustering Algorithms for Wireless Sensor Networks", Journal of Computer Communications, Vol 30, No. 13, 2007, pp. 28 26- 2841.

[2] M. BaniYassein, A. Al-zou'bi, Y. Khamayseh, W.Mardini, "An Improvement on LEACH Protocol of Wireless Sensor Network (VLEACH)," International Journal of Digital Content Technology and its Applications Volume 3, Number 2, June 2009.

[3] Lindsey, S.; Raghavendra, C.; Sivalingam, K.M. Data gathering algorithms in sensor networks using energy metrics. IEEE Trans. Parallel Distrib. Syst. 2002, 13, 924-935.
[4] Koutsonikola, D.; Das, S.; Charlie, H.Y.; Stojmenovic, I. Hierarchical geographic multicast routing for wireless sensor networks. Wirel. Netw. 2010, 16, 449-466.

[5] Hari, U., B. Ramachandran, and Chris Johnson. "An Unequally Clustered Multihop Routing protocol for Wireless Sensor Networks." In Advances in Computing, Communications and Informatics (ICACCI), 2013 International Conference on, pp. 1007-1011. IEEE, 2013.

[6] S. Banerjee and S. Khuller, A clustering scheme for hierarchical control in multi-hop wireless networks, in Proceedings of 20th Joint Conference of the IEEE Computer and Communications Societies (INFOCOMŠ 01), Anchorage, AK, April 2001.

[7] Smaragdakis, Georgios, Ibrahim Matta, and AzerBestavros. "SEP: A stable election protocol for clustered heterogeneous wireless sensor networks", Boston University Computer Science Department, 2004.

[8] Femi A. Aderohunmu, Jeremiah D. Deng,“ An Enhanced Stable Election Protocol for Clustered Heterogeneous WSN".

[9] Li Qing, Qingxin Zhu, Mingwen Wang, "Design of a distributed energy-efficient clustering algorithm for heterogeneous wireless sensor networks ", Computer Communications, Volume 29, Issue 12, 4 August 2006, Pages 2230-2237.

[10] Rehman O., Nadeem Javaid, Basit Manzoor, A. Hafeez, Adeel Iqbal, and M. Ishfaq. "Energy Consumption Rate based Stable Election Protocol (ECRSEP) for WSNs." Procedia Computer Science 19 (2013): 932-937.

[11] Mamalis, Basilis, Damianos Gavalas, Charalampos Konstantopoulos, and Grammati Pantziou. "Clustering in wireless sensor networks." RFID and Sensor Networks: Architectures, Protocols, Security and Integrations, Y. Zhang, LT Yang, J. Chen, eds (2009): 324-353.

[12] Manjeshwar, E.; Agrawal, D.P. TEEN: A Routing Protocol for Enhanced Efficiency in Wireless Sensor Networks. In Proceedings of the 15th International Parallel and Distributed Processing Symposium (IPDPS), San Francisco, CA, USA, 23-27 April 2001; pp. 20092015. 